International Diagnostic Interview (CIDI), lacks sensitivity because of the strict diagnostic rule. This could account for the low prevalence rate in our study and the fact that we may have picked up only those most seriously affected. However, we do not doubt the presence of depression in our society. The impetus for our study came from the 'lived lives' of Ethiopian psychiatrists working within Ethiopia who commonly encounter people presenting with symptoms according to a 'Western' construct of depression in a tertiary care setting. These people respond to antidepressants by showing good recovery from symptoms and regaining their original level of functionality. In an ongoing intervention programme, we have found the same for people with depression identified by the CIDI in Butajira (study ongoing).

The CIDI was translated, back-translated and modified by experienced Ethiopian psychiatrists who considered the symptom questions to have face validity and applicability. In addition, convergent validity of CIDI-defined depression was indicated by our finding of strong associations between depression and disability. We believe that the CIDI is unlikely to be merely detecting physical ill health because first, it incorporates specific measures to screen out symptoms that seem to have a physical cause and second, our study participants with persistent depression were most disabled in social domains rather than in those domains of functioning more likely to be influenced by physical impairment (e.g. mobility).

We believe that the difference in mental health across cultures is mainly in the presenting features, not in the nature of the disorder. In low- and middle-income countries it has been said that people tend to present with somatic symptoms (Mumford et al, 1997; Parker et al, 2001). However, this view of cultural difference between the West and the rest of the world was challenged by the World Health Organization cross-cultural study in primary care (Gureje et al, 1997). Although the presentation of depression clearly does vary across cultures, in an African setting depression was found to be better characterised by core depressive symptoms than by somatic complaints (Okulate et al, 2004).

Gureje, O., Simon, G. E., Ustun, T. B., et al (1997) Somatization in cross-cultural perspective: a World Health Organization study in primary care. American Journal of Psychiatry, 154, 989-995.
Mumford, D. B., Saeed, K., Ahmad, I., et al (1997) Stress and psychiatric disorder in rural Punjab. A community survey. British Journal of Psychiatry, 170 473-478.

Okulate, G. T., Olayinka, M. O. \& Jones, O. B. E. (2004) Somatic symptoms in depression: evaluation of their diagnostic weight in an African setting. British Journal of Psychiatry, 184, 422-427.

Parker, G., Gladstone, G. \& Chee, K. T. (200I) Depression in the planet's largest ethnic group: the Chinese. American Journal of Psychiatry, 158, 857-864.

S. Mogga, R. Stewart Institute of Psychiatry, De Crespigny Park, PO60, London SE5 8AF, UK. Email: S.frissa@iop.kcl.ac.uk

doi: 10.I192/bjp.190.4.362b

\section{Suicide risk and perinatal circumstances}

Riordan et al (2006) present evidence that maternal circumstances and foetal experience may have an impact upon the subsequent mental health of the offspring. Many studies describe gestational insults, obstetric complications and perinatal environment as risk factors for mental illness in later life. Such evidence has often been based upon longitudinal cohort studies which have the advantage of large sample sizes and masked assessments at both exposure and outcome. Such strategies have generated evidence supporting the neurodevelopmental hypothesis of schizophrenia (Done et al, 1991; Jones et al, 1994). There can be little doubt that such epidemiological evidence can lead to hypotheses of the pathogenesis of psychiatric illness. As suggested by Riordan et al, foetal nutrition, intra-uterine stressors, hypothalamicpituitary-adrenal axis dysfunction and attachment theory may all be putative mechanisms by which the foetal-maternal interaction contributes to future psychiatric illness.

However, there are several inherent limitations to this methodology. Longitudinal studies have traditionally concentrated on descriptions of the progeny. Data relating to many maternal factors in birth cohort studies are limited or unavailable. Important confounding factors cannot be, and have not been, eliminated in such work. Social class, alluded to by Riordan et al, cannot be ignored as an important confounder for all of the findings. Riordan et al concede that assessment of economic circumstances is based only on parental occupation. Is such a measure valid over several generations? How can social class (a factor relating both to parity, and to young maternal age and depression and suicide) not be considered an important confounding variable? The importance of housing and maternal diet, alcohol, smoking and drug use will certainly influence birth weight and depression and suicide in later life. Family history of psychiatric illness is probably the most important confounding factor that has not been, and unfortunately cannot be, assessed in this study. Maternal depression may have an impact upon birth weight as an environmental factor, but may exert a genetic effect on psychiatric illness and suicide of the offspring. Although such a study does suggest important epidemiological trends, hypothesising that biological mechanisms are involved in psychiatric illness of offspring is premature without controlling for these important confounders.

Done, D. J., Johnstone, E. C., Frith, C. D., et al (1991) Complications of pregnancy and delivery in relation to psychosis in adult life: data from the British perinatal mortality survey sample. BMJ, 302, 1576-1580.

Jones, P., Rodgers, B., Murray, R., et al (1994) Child development risk factors for adult schizophrenia in the British 1946 birth cohort. Lancet, 344, 1398-1402.

Riordan, D.V., Selvaraj, S., Stark, C., et al (2006) Perinatal circumstances and risk of offspring suicide: birth cohort study. British Journal of Psychiatry, 189, 502507.

B. J. Baig Division of Psychiatry, University of Edinburgh, Royal Edinburgh Hospital, Edinburgh, UK. Email: bbaig@staffmail.ed.ac.uk

doi: 10.I192/bjp.190.4.363

Authors' reply: Dr Baig may be correct in reiterating the inherent weaknesses of longitudinal birth cohort studies, but we do not accept that it is premature to hypothesise. Future studies into these potentially important epidemiological trends will require modified study designs and therefore hypotheses to guide these. We have discussed a heterogeneous group of potential confounding and mediating factors, biological influences being just one possible aspect of what is probably a complex picture of multifactorial aetiology. Hypothesising about the exclusive involvement of biological factors would indeed be premature, but not to consider them at all would place undue restrictions on future study design.

D.V. Riordan, C. Stark, S. Selvaraj New

Craigs Hospital, 6-16 Leachkin Road, Inverness IV3 8NP, UK. Email: Vincent.Riordan@haht.scot.nhs.uk doi: 10.1192/bjp.190.4.363a 